Article

\title{
A Novel Stilbene-Like Compound That Reduces Melanin through Inhibiting Melanocyte Differentiation and Proliferation without Inhibiting Tyrosinase
}

\author{
Kristy Martinson ${ }^{1}$, Noah Stueven ${ }^{1}$, Aaron Monte ${ }^{2}$ and Cheng-chen Huang ${ }^{1, *}$ \\ 1 Biology Department, University of Wisconsin-River Falls, River Falls, WI 54022, USA; \\ kjmartinson@wisc.edu (K.M.); noah.stueven@my.uwrf.edu (N.S.) \\ 2 Department of Chemistry and Biochemistry, University of Wisconsin-La Crosse, La Crosse, WI 54601, USA; \\ amonte@uwlax.edu \\ * Correspondence: cheng-chen.huang@uwrf.edu; Tel.: +715-425-4276; Fax: 715-425-0378
}

Received: 24 May 2018; Accepted: 11 July 2018; Published: 19 July 2018

\begin{abstract}
Cosmetic practices that use skin-lightening agents to obtain desired skin tones or treat pigment abnormalities have been popular worldwide. However, the molecular and cellular mechanisms of these agents are still largely unknown. Here we identified a family of compounds, with the lead compound named A11, that exhibited strong pigment reduction in developing zebrafish embryos. The pigment inhibition lasted for several days and is effective both before and after melanogenesis. By comparison with several known skin-lightening compounds, A11 appeared to be more potent and caused slower pigment recovery after withdrawal. A11, however, did not inhibit tyrosinase or cause apoptosis in melanocytes. We further found that A11 suppressed proliferation in melanocytes and reduced the number of differentiated melanocytes by activating MAPK (mitogen-activated protein kinase) and Akt. Finally, A11 also caused melanin reduction in mammalian melanocytes. Together, A11 might be a potent skin-lightening agent with novel mechanisms.
\end{abstract}

Keywords: skin-lightening; zebrafish; tyrosinase; melanocyte; proliferation; differentiation

\section{Introduction}

The desire to manipulate skin color has long been observed in human history. It is well known that melanin, the pigment molecule that determines our skin color, plays an important role in absorbing high energy waves, such as ultraviolet light, to protect molecules like DNA from damages induced by the energy wave. Damages can include abnormal chemical bonding formation and DNA breaks which can eventually lead to cancer formation. It is well documented that people with fair skin color appear to have a significantly higher risk of skin cancer. This explains why darker (or tanned) skin is believed to be healthier and preferred in Caucasians. In contrast, people in African and Asian countries prefer a lighter skin color because it is commonly associated with higher social status. Therefore, while the sunbath and tanning industry is popular in western countries, skin-lightening products and practices are sought after in Asia.

Skin color can easily and quickly be changed due to the high turnover rate of skin cells and the simple enzymatic pathway for melanin synthesis. Keratinocytes make up approximately $90 \%$ of our epidermis and are responsible for even skin tone. Keratinocytes are constantly exposed to environmental toxins and damages causing a high turnover rate. It is estimated that the turnover rate ranges from two weeks in infants to one month for people in their thirties. Melanin pigment molecules are synthesized in melanocytes, which only make up $1 \%$ of epidermis, and later are transported 
to keratinocytes. The biochemical synthesis pathway for melanin starts with tyrosinse amino acid catalyzed by tyrosinase into $\beta$-3,4-dihydroxyphenylalanine (DOPA) and later into DOPAquinone. Thus tyrosinase is the key enzyme of melanin synthesis. There are many naturally found tyrosinase inhibitors that can effectively reduce pigment both in vitro and in vivo. For example, kojic acid isolated from Aspergillus and Penicillum species is a strong tyrosinase inhibitor that chelates copper which is a cofactor of tyrosinase (Battaini et al. [1]). Arbutin, derived from the fruit of the California buckeye, is another strong tyrosinase inhibitor while aloesin, a natural derivative from aloe vera, acts as a competitive inhibitor of tyrosinase (Jones et al. [2]). Some other pigment reduction reagents were found to function during melanin transport or in regulating melanocyte survival (Reviewed in Kim et al. [3]). Because of the high turnover rate of keratinocytes, topical application of these reagents can easily generate lighter skin color within a few weeks. However, with the understanding of melanogenesis mechanisms, it is also possible to increase skin pigmentation (Brenner and Hearing [4]).

Adverse effects of skin-lightening agents have been reported, although it is commonly considered safe toward ectopically applied compounds. Hydroquinone, which was the very first skin-lightening compound, is a strong tyrosinase inhibitor. Its specificity didn't raise any concern when it was first used on humans, but later was found to cause cytotoxicity in melanocytes, DNA damages, and likely cancers (Eastmond et al. [5]). Derivatives of hydroquinone, the natural form called ${ }^{\circledR}$-arbutin and the synthetic form $\alpha$-arbutin, exhibit strong tyrosinase inhibition but with no detectable cytotoxicity. No detailed toxicity study has been conducted for arbutin derivatives. Kojic acid, another commonly used skin-lightening agent, has raised concerns about potentially being a carcinogen (Takizawa et al. [6]). Tretinoin, a precursor of vitamin A which is effective in treating hyperpigmentation, was found to cause erythema and peeling due to its function in accelerating epidermal turnover (Mezick et al. [7]). More recently, a new product, rhododendrol from Acer nikoense and Betula platyphylla developed by Kanebo, Inc. in Japan had to be withdrawn from market because of its unexpected result of vitiligo-like skin disorder (Sasaki et al. [8]). To date, the list of skin-lightening agents continues growing rapidly but only a handful of them have been carefully characterized in their toxicity and adverse effect.

A number of skin-lightening agents seem to regulate intracellular signaling pathways, which leads to a decrease of melanin synthesis, and/or increase of melanocyte cell death, and/or possibly melanocyte differentiation. Although the current understanding is that activation of MAPK and/or Akt signaling pathways can lead to pigment increase (Imokawa and Ishida [9]), several new skin-lightening agents have begun to show exceptions. For example, haginin A, a recently identified tyrosinase inhibitor from Lespedeza cyrtobotrya, functions as a noncompetitive inhibitor of tyrosinase but also induces the MAPK and Akt signaling pathways (Kim et al. [10]). Another study showed, however, the extract from Rhodobacter sphaeroides inhibits melanin formation by inhibiting MAPK but activating Akt pathways (Liu et al. [11]). Yet gallic acid was shown to inhibit melanogenesis by activating both MAPK and Akt signaling pathways (Su et al. [12]). Therefore, it is still not yet conclusively known how MAPK and Akt are involved in melanogenesis.

Zebrafish are a very popular tool for basic studies on pigment and color pattern formation due to the highly conserved mechanisms of melanocyte development and melanin synthesis between fish and human (Irion et al. [13]). The fast development of melanocytes and ease of observing pigment change in zebrafish embryos makes it an ideal assay system for testing compounds that are potential skin-lightening agents (Choi et al. [14]). The zebrafish embryo is also an excellent vertebrate model for toxicity evaluation for potential drugs (Garcia et al. [15]). However, at least one fundamental difference in skin color formation exists between fish and mammals. Unlike in mammals, where melanin is transported from melanocytes to keratinocytes through melanosomes, zebrafish do not have a melanosome transportation process (Irion et al. [13]), therefore compounds that show an effect on zebrafish pigment are primarily affecting melanin synthesis and/or metabolism or melanocyte biology.

Our lab has been using an established zebrafish heart failure model (Huang et al. [16]) to look for compounds that could attenuate heart failure progression. Among the several positive compounds that were identified in the last few years, A11 and MEK-I were also able to cause significant 
reduction of black pigment in the zebrafish embryos. Since MEK-I is a known inhibitor of the MEK (mitogen-activated protein kinase kinase) which functions in the MAPK pathway to promote melanin synthesis (reviewed in Imokawa and Ishida [9]), it is not so surprising to see MEK-I causing pigment reduction. A11, however, has a very different chemical structure than MEK-I and belongs to the stilbene family which has a characteristic C6-C2-C6 structure, similar to that in resveratrol (Figure 1). In this article, we report the skin-lightening effect of MEK-I and A11. Our in vivo studies using zebrafish embryos revealed the differential pharmacodynamics of these two compounds. Unlike most stilbenoids, A11 is not a tyrosinase inhibitor. Through cellular and molecular characterizations, we found that A11 seems to have a novel mechanism than other skin-lightening compounds in inhibiting pigment formation by potentially regulating melanocyte differentiation and proliferation (more supporting data can be found in Stueven et al. [17]).

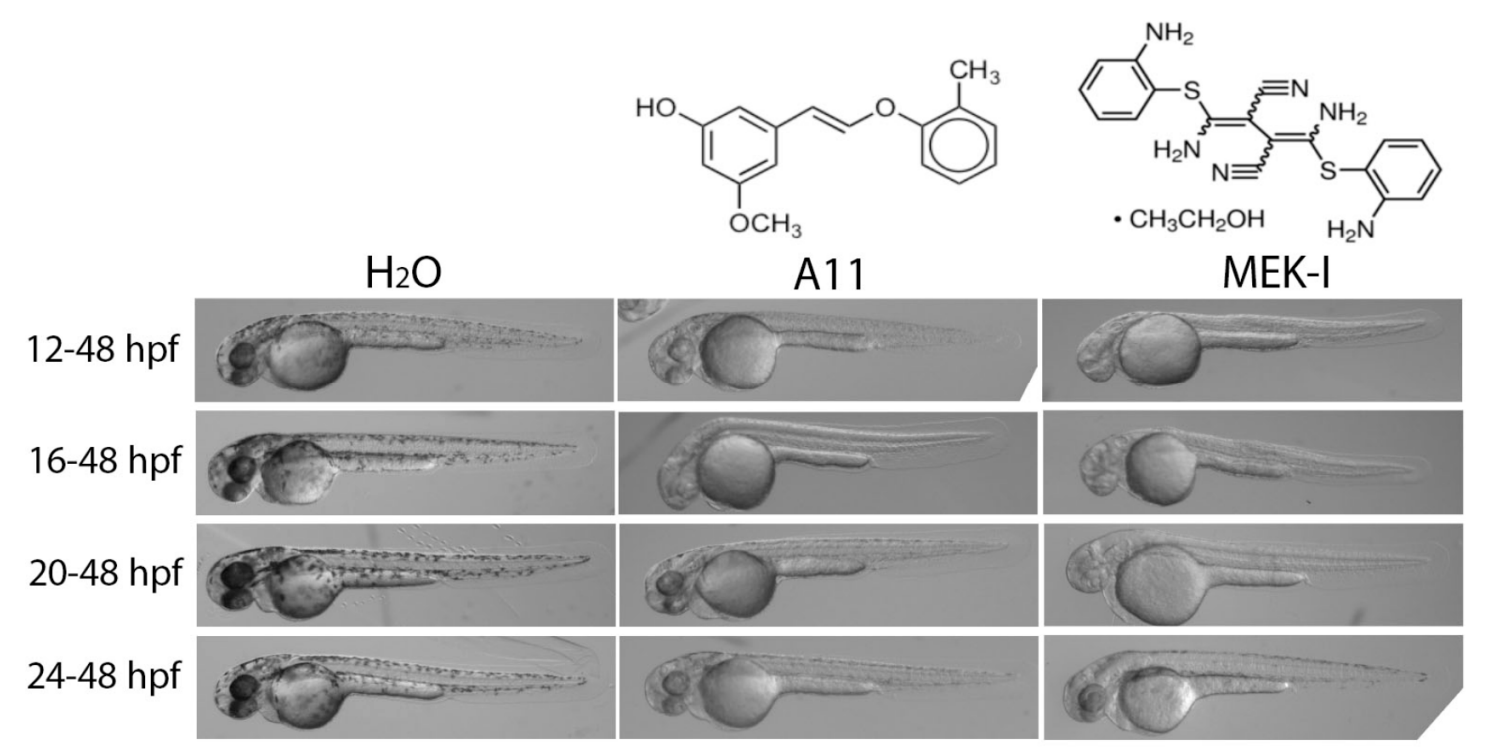

Figure 1. A11 and MEK-I cause strong pigment inhibition in early zebrafish embryos. Zebrafish embryos were treated with A11 or MEK-I starting from 12, 16, 20, or 24 hpf (hours post fertilization) to 48 hpf. Embryos in $\mathrm{H}_{2} \mathrm{O}$ showed normal level of pigment. A11 and MEK-I both caused strong inhibition in pigment formation. However, while A11 showed consistent potency in the different stages of treatment, the potency of MEK-I appeared weaker in the $24-48 \mathrm{hpf}$ embryo.

\section{Materials and Methods}

\subsection{Zebrafish Husbandry and In Vitro Fertilization}

The zebrafish stocks used in this study are maintained following standard procedures (Westerfield [18]) and bred by in vitro fertilization. In brief, mature male and female zebrafish were separated by a mesh in a breeding tank the night before breeding. Soon after the light was turned on the next morning, fish were anesthetized in $\sim 0.16 \%$ tricaine for $1-2 \mathrm{~min}$. The females were placed on a paper towel to dry the body surface briefly before being transferred into a $6 \mathrm{~cm}$ dish. The eggs were expelled onto the dish by gently pressing the latero-ventral side of the belly with fingers. The males were also dried briefly and then placed ventral side up on a sponge well. Under a dissection microscope, the lateral sides of the belly were pressed gently with a pair of blunt forceps while holding a capillary tube close to the anus to collect sperm. The sperm were immediately mixed with the eggs and $\sim 1 \mathrm{~mL}$ of egg water was added (more details in Westerfield [18]). 


\subsection{Chemical Treatment of Zebrafish Embryos}

Zebrafish embryos were collected and arrayed 5 embryos per well of a 96-well plate in $200 \mu \mathrm{L}$ of egg water (distilled water containing $60 \mu \mathrm{g} / \mathrm{mL}$ sea salt from Coralife, CA, USA) which were later replaced with the same volume of egg water containing the desired compound. In the recovery experiment, embryos were set up in a 96-well plate with the compound from $24-72 \mathrm{hpf}$, after which they were transferred to a $6 \mathrm{~cm}$ dish to be washed three times with egg water and allowed to continue their development for several days. Pigment analyses were done using wild type embryos as $100 \%$ pigment and visually comparing the pigment amount in compound-treated embryos. We typically set up 20 embryos for each treatment group in each experiment with repetition of each experiment.

\subsection{Chemicals}

A11 and its analogs were synthesized by Dr. Monte at UW-La Crosse. Other chemicals used in this study were purchased from Sigma-Aldrich (St. Louis, MI, USA): MEK-I (U0126), MoTP (4-(4-Morpholinobutylthio)phenol, SML0047), and phenoxodiol (D7446) were all prepared at $10 \mathrm{mM}$ in DMSO, arbutin (A4256) was at $300 \mathrm{mM}$, kojic acid (K3120) at $10 \mathrm{mM}$, niacinamide (N5535) $20 \%(w / v)$, tretinoin (PHR1187) 10\% (w/v), and PTU (phenylthiourea, P7629) 0.3\% (w/v) all in ddH2O.

\subsection{In Vitro Tyrosinase Assay}

The drug effect on tyrosinase was measured by the in vitro tyrosinase assay (Baurin et al. [19]). Purified mushroom tyrosinase (cat. T3824, $2100 \mathrm{Unit} / \mathrm{mL}$ ) and L-tyrosine (T3754, $1.5 \mathrm{mM}$ ) were purchased from Sigma-Aldrich. The reactions were set up in triplicate in a 96-well plate. Each reaction contained $30 \mu \mathrm{L}$ L-tyrosine with $20 \mu \mathrm{L}$ of tyrosinase and $130 \mu \mathrm{L}$ of $0.1 \mathrm{M}$ phosphate buffer $\mathrm{pH} 6.5$ with or without the test compound $(20 \mu \mathrm{L})$. The plate was then taken to a microplate reader (Bio-Rad Model 680 ) to measure the absorbance at $475 \mathrm{~nm}$ at multiple time points.

\subsection{Immunohistochemistry and TUNEL Assay}

Embryos were fixed in $4 \%$ paraformaldehyde at least overnight in $4{ }^{\circ} \mathrm{C}$. The fixed embryos were washed twice with PBS, (phosphate buffered saline) then once with $\mathrm{H}_{2} \mathrm{O}, 5 \mathrm{~min} / \mathrm{each}$, and permeabilized with $-20{ }^{\circ} \mathrm{C}$ acetone for $7 \mathrm{~min}$ followed by washes in $\mathrm{H}_{2} \mathrm{O}$ and then PBS. The embryos were incubated with 3\% BSA (bovine serum albumin) in PBST (PBS with $0.1 \%$ Tween 20) for at least $1 \mathrm{~h}$ at room temperature before overnight incubation in $4{ }^{\circ} \mathrm{C}$ with the phosphor-Histone H3 (Ser10) Rabbit mAb-Alexa Fluor 555 conjugate in PBST (Cell Signaling Technology). The next day, the embryos were washed with PBST at least 4 times for $15 \mathrm{~min} /$ each, then stored in ProLong Gold Antifade Reagent with DAPI (Cell Signaling Technology, Danvers, MA, USA). Apoptotic cells were detected by TUNEL assay using the TMR In Situ Cell Death Detection Kit from Roche.

\subsection{In Situ Hybridization}

In situ hybridization protocol was adopted from The Zebrafish Book (Westerfield [18]). Because of the concern that our compounds do not cause complete albino and the residual pigment might interfere with the in situ hybridization signal and skew our analysis, we treated embryos with desired compounds with PTU, and those embryos treated with PTU alone were used as a positive control in this experiment. PTU does not cause cell death or developmental defects in melanocyte. Twenty embryos were set up for each compound and treated from $24-48 \mathrm{hpf}$ then fixed for in situ hybridization.

\subsection{Western Blotting}

Twenty embryos were first treated with desired compound from $24-48 \mathrm{hpf}$ followed by pigment analysis using a dissection microscope. Embryos were then de-chorionized and put into $200 \mu \mathrm{L}$ of lysis buffer $(0.2 \% \mathrm{NP}-40,100 \mathrm{mM}$ Tris, $150 \mathrm{mM} \mathrm{NaCl}, 8 \mathrm{mM}$ of EDTA, pH 7.4) with protease inhibitor cocktail added freshly (P8340, Sigma). Embryos were grinded with plastic pestles for 5 min 
and then further lysed for $2 \mathrm{~h}$ in $4{ }^{\circ} \mathrm{C}$ on a shaker. The lysate was then centrifuged at 12,000 rpm, $4{ }^{\circ} \mathrm{C}$, for $20 \mathrm{~min}$. The supernatant was transferred to a clean tube and stored in $-80{ }^{\circ} \mathrm{C}$. Protein concentration was determined using the Pierce BCA Assay Kit (23227, Thermo Scientific, Waltham, MA, USA). 10-20 $\mu \mathrm{g}$ of proteins were loaded into 12\% SDS-PAGE gel (Bio-Rad) for gel electrophoresis and later for Western blotting using the Pierce Fast Western Blot Kit (35050, Thermo Scientific, Waltham, MA, USA). Antibodies were purchased from Cell Signaling Technology.

\subsection{Cell Culture}

The mouse melanoma cell line B16F10 (ATCC CRL-6475) was purchased from ATCC (American Tissue Culture Center, Manassas, VA, USA) and maintained in DMEM (Dulbecco/Vogt modified Eagle's minimal essential medium) supplemented with $10 \%$ fetal bovine serum. The human primary epidermal melanocytes (PCS-200-013) was also purchased from ATCC and maintained in Dermal Cell Basal Medium (PCS-200-030) supplemented with the Adult Melanocyte Growth Kit (PCS-200-042). Trypsinization and subculture were performed following the protocols found on the ATCC website. For chemical treatment, approximately 14,000 melanoma cells and 30,000 normal melanocytes were seeded in each well of the 24 -well plate with $0.5 \mathrm{~mL}$ of culture medium. Cells were set up in triplicate and allowed to grow two days for melanoma or three days for normal melanocytes before chemical treatment. After $24 \mathrm{~h}$ of chemical incubation, cells were lysed with $250 \mu \mathrm{L}$ of $1 \mathrm{~N} \mathrm{KOH}$ in the well and $180 \mu \mathrm{L}$ of lysate was transferred to a flat-bottom 96-well plate for melanin measurement.

\section{Results}

\subsection{A11 and MEK-I as Skin-Lightening Compounds}

To understand the pharmacodynamics of A11 and MEK-I, we characterized the two compounds in a series of experiments using zebrafish embryos. First, we tested how early the compounds are required to produce pigment inhibition phenotypes in the developing embryos. Embryos were treated with A11 and MEK-I at different stages, 12, 16, 20, $24 \mathrm{hpf}$ (hours post fertilization) and examined for pigment development at $48 \mathrm{hpf}$. While A11 caused similar degrees of pigment inhibition in these embryos, MEK-I showed complete inhibition in early embryos but less inhibition in 24 hpf embryos (Figure 1). MEK-I appeared to have no inhibition at all in the $30 \mathrm{hpf}$ embryos (data not shown), indicating MEK-I only functions in the early stage of pigment formation. Alternatively, MEK-I might have a slower drug effect so that the inhibition was not apparent within the 16-h (from 30-48 hpf) treatment. This was proved not to be the case as we saw no pigment inhibition by MEK-I at later time points (data not shown).

Next, we tested the duration of drug effect. Embryos at $24 \mathrm{hpf}$ stage were treated with MEK-I or A11 and observed at 48 and 72 hpf. Embryos treated with A11 showed about the same pigment inhibition at both 48 and $72 \mathrm{hpf}$ stages but those in MEK-I only showed effective pigment reduction in 48 but not in 72 hpf embryos (Figure 2A), indicating A11 has longer inhibition ability than MEK-I. Embryos incubated in MEK-I from 12-72 hpf showed almost normal level of black pigment, but those incubated from 20-72 hpf showed greatly reduced pigment content (Figure 2B), indicating the effective duration of MEK-I is approximately $50 \mathrm{~h}$ long. By contrast, A11 caused similar level of pigment reduction in embryos at all the stages tested, ranging from 14-72 hpf and the pigmentation inhibition lasted at least for 4 days (data not shown). Thus, A11 has a broader time window and longer skin-lightening effect than MEK-I. All these results suggest that A11 and MEK-I have different inhibitory mechanisms. 


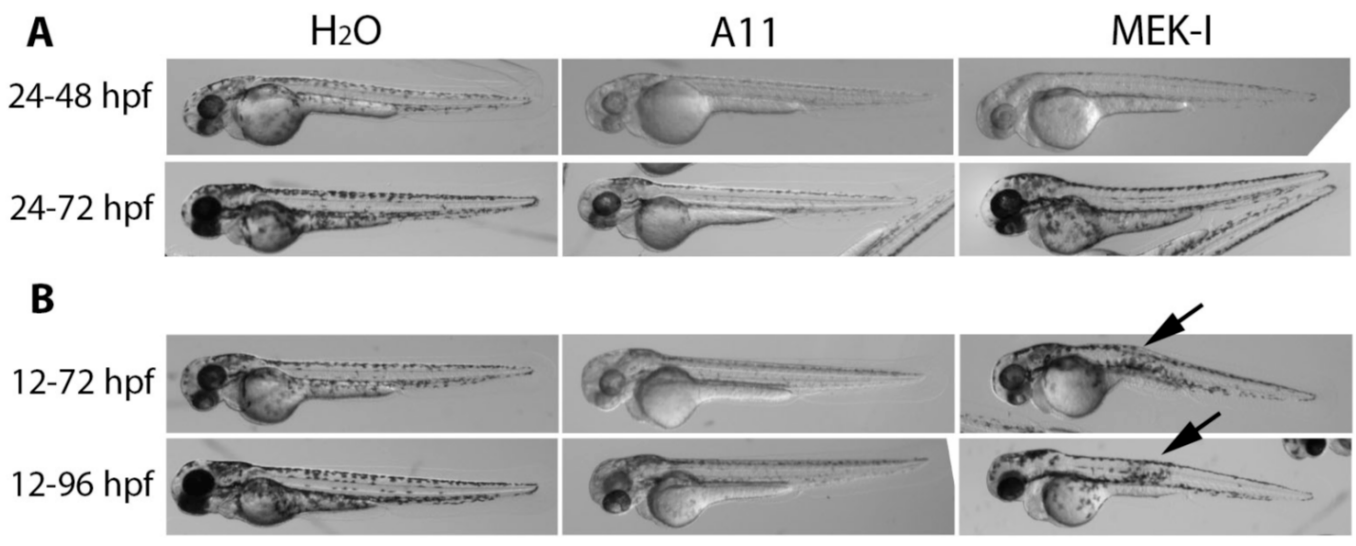

Figure 2. Differential pharmacodynamics of A11 and MEK-I. (A) Embryos were treated with A11 or MEK-I for different lengths of time. A11 maintained fairly strong pigment inhibition from $24-48$ hpf to 24-72 hpf while MEK-I apparently lost its potency in the 24-72 hpf embryo, compared to the MEK-I 24-48 hpf embryo; (B) When embryos were treated from $12 \mathrm{hpf}$ for 3-4 days, A11 still maintained strong pigment inhibition but MEK-I did not show any inhibition in the 12-72 or 12-96 hpf embryos. Arrows point to the notochord defects caused by MEK-I.

\subsection{Stronger Potency of A11 over Most Skin-Lightening Compounds}

Zebrafish embryos have been used to test skin-lightening compounds due to its conserved melanin development mechanisms (Dooley et al. [20]). The popular human skin-lightening compounds arbutin and kojic acid had been shown to produce strong skin-lightening effect on developing zebrafish embryos (Choi et al. [14]). We next compared A11 and MEK-I with several human skin-lightening compounds that are currently on the market: arbutin, kojic acid, niacinamide, tretinoin, and two recently reported compounds, haginin A (Kim et al. [10], also called phenoxidiol) and MoTP (Yang et al. [21]) as well as the well-known tyrosinase inhibitor, phenylthiourea (PTU). We found that these skin-lightening compounds caused different degrees of pigment reduction in zebrafish embryos (Figure 3A). In summary, A11, arbutin, MoTP, phenoxodiol, and PTU could reliably reduce the pigment by approximately $90-100 \%$. MEK-I had the same effect but only when it is applied to younger embryos. Staying consistent with its known tyrosinase inhibitory activity, kojic acid caused a lighter color in all the melanocytes (Figure 3C). These comparison studies validate the potential skin-lightening effect of A11 and MEK-I on humans.

In the previous experiment, we also noted that several human skin-lightening compounds appeared to cause toxicity in developing embryos, most of which have not been reported before. In arbutin-treated embryos, we observed cardiac arrest within 2-4 days of incubation in a dosage dependent manner. Unlike most cardiac toxins which commonly cause cardiac edema, there was no dramatic morphological defects or cardiac edema in the arbutin-treated embryos (unpublished results). Niacinamide, phenoxodiol, and tretinoin appeared to disrupt other developmental processes causing severe morphological defects in multiple tissues (Figure 3A). MoTP has been shown to cause melanocyte cell death in a tyrosinase-dependent manner (Yang et al. [21]). MEK-I is interesting in that it caused notochord development defects (Figure 1, Hawkins et al. [22], Huang et al. [16]), but only in early embryos. PTU also cause notochord distortion in early embryos, presumably due to its ability to chelate copper ions (also reported in Hawkins et al. [22]. Older embryos treated with MEK-I or PTU were able to develop normal notochords and live (Figures 1 and 3). 


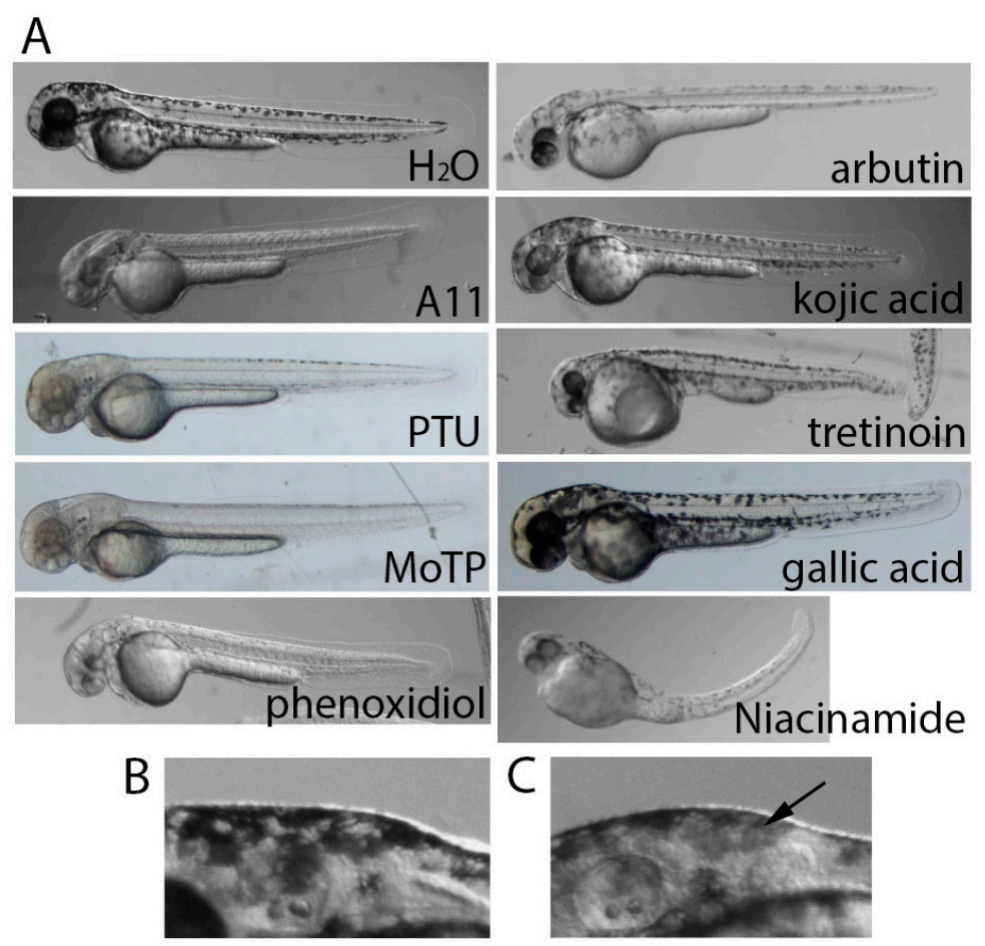

Figure 3. Comparison between A11 and known skin-lightening compounds. (A) Zebrafish embryos were treated with different skin-lightening compounds to show different degrees of pigment inhibition. A11 $(10 \mu \mathrm{M})$, PTU $(0.003 \%)$, MoTP $(10 \mu \mathrm{M})$, and phenoxidiol $(10 \mu \mathrm{M})$ all exhibited strong pigment inhibition, but ${ }^{\circledR}$-arbutin $(100 \mathrm{mM})$, kojic acid $(10 \mathrm{mM})$, tretinoin $(1 \%)$, gallic acid $(1 \mathrm{mM})$, and niacinamide (1\%) showed weaker inhibition. Some skin-lightening compounds apparently caused strong toxicity to developing embryos, body curvature by niacinamide and eye and tail defects by tretinoin. High magnification of the head area of embryos in $\mathrm{H}_{2} \mathrm{O}(\mathbf{B})$ and kojic acid (C) are shown to display the lighter pigment in melanocytes (arrow) caused by kojic acid.

\subsection{A11 Reduces Existing Pigment}

To test whether the skin-lightening compounds had any effect on already formed pigment, we treated $48 \mathrm{hpf}$ embryos with the compounds until 72 or $96 \mathrm{hpf}$. Interestingly, while most skin-lightening compounds had little or no effect on existing pigment, A11 caused significant reduction of pigment within $24 \mathrm{~h}$ of treatment (Figure $4 \mathrm{~A}$ ). Interestingly, the melanocytes in A11-treated embryos appeared contracted than those in control (Figure 4C).

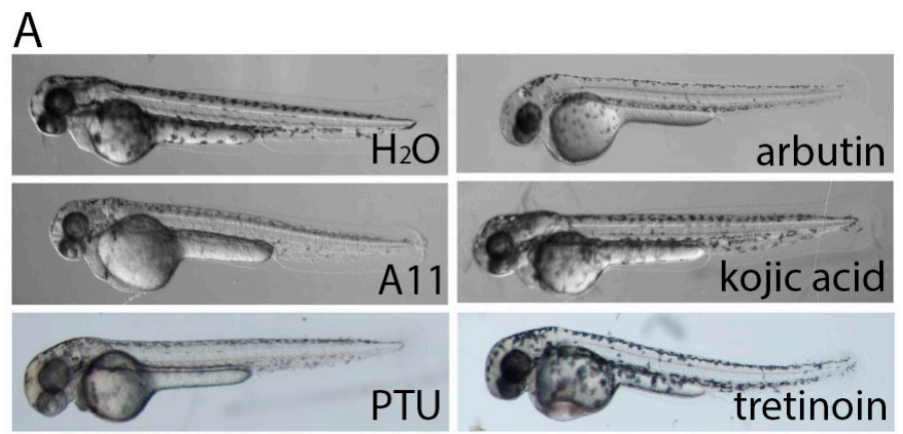

Figure 4. Cont. 

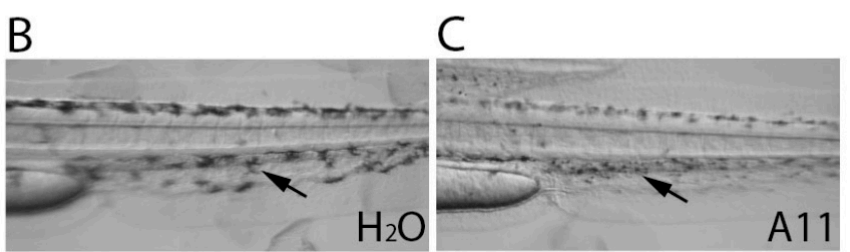

Figure 4. A11 reduces existing pigment. (A) Embryos at $48 \mathrm{hpf}$ stage that already generated lots of pigment were selected for this experiment to receive compound treatment until $72 \mathrm{hpf}$ and then were photographed. A11 caused significant pigment reduction compared to the control at $72 \mathrm{hpf}$ (even less than 48 hpf normal embryo in Figure 3). High magnification of $\mathrm{H}_{2} \mathrm{O}(\mathbf{B})$ and $\mathrm{A} 11$ (C) treated embryos are shown to display the contracted morphology of melanocytes (arrows).

\subsection{Slow Pigment Recovery by A11}

As most skin-lightening compounds are tyrosinase inhibitors, it has been shown that pigmentation could recover within $24 \mathrm{~h}$ after compounds were washed off. To test this notion, embryos were treated with skin-lightening compounds from $20-48$ or $72 \mathrm{hpf}$, washed twice, and transferred to egg water for $24 \mathrm{~h}$ or longer. The results showed that pigmentation in most embryos did recover quickly but not in A11 or arbutin-treated embryos (Figure 5B).

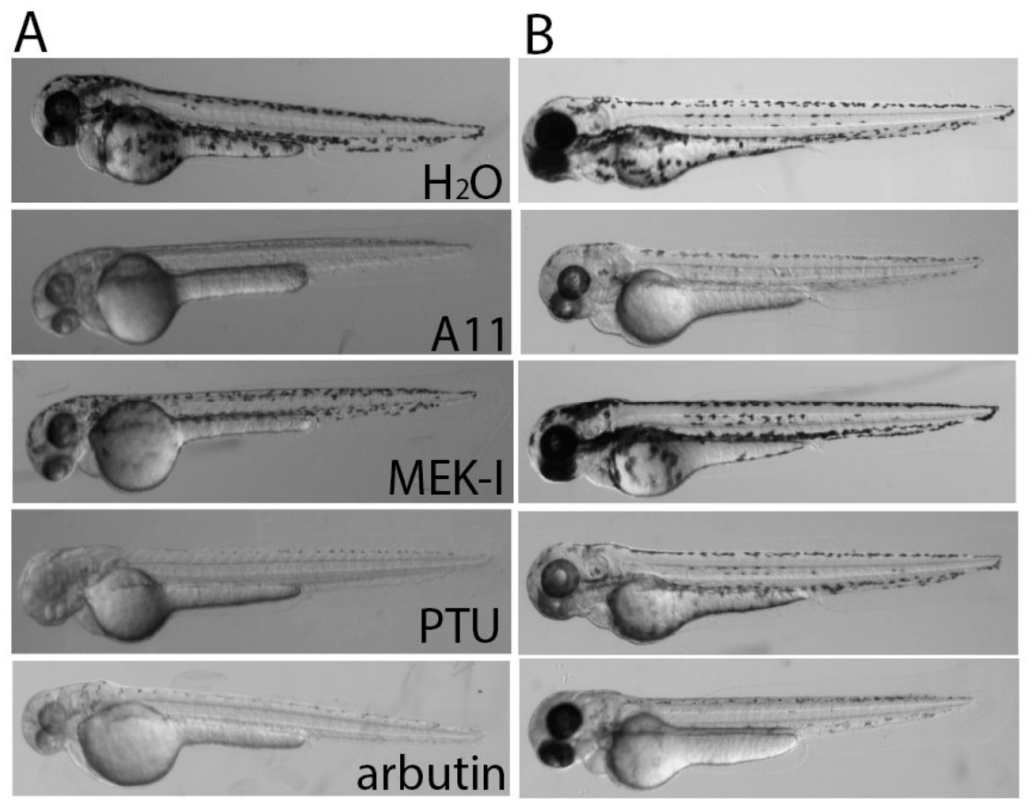

Figure 5. Slow pigment recovery by A11. Embryos were treated with skin-lightening compounds from 24-72 hpf (A) then washed with egg water twice and allowed to develop until $96 \mathrm{hpf}(\mathbf{B})$ in water to test how quickly pigment recovers after compound withdrawal. Most embryos recovered their pigment quickly except for A11 and arbutin.

\subsection{A11 is Not a Tyrosinase Inhibitor}

It has been shown that many stilbenoids have tyrosinase inhibitory activity (Likhitwitayawuid [23]). Since A11 is a stilbene-like compound, we tested whether A11 or MEK-I can inhibit tyrosinase using an in vitro tyrosinase assay (Baurin et al. [19]). While most of the skin-lightening compounds showed strong inhibition of tyrosinase, neither A11 or MEK-I did at either $10 \mu \mathrm{M}$ and $50 \mu \mathrm{M}$ (Figure 6 and data not shown). It is important to note that phenoxodiol had a very short tyrosinase inhibition efficacy but gallic acid and MoTP actually enhanced tyrosinase activity (Figure 6). 


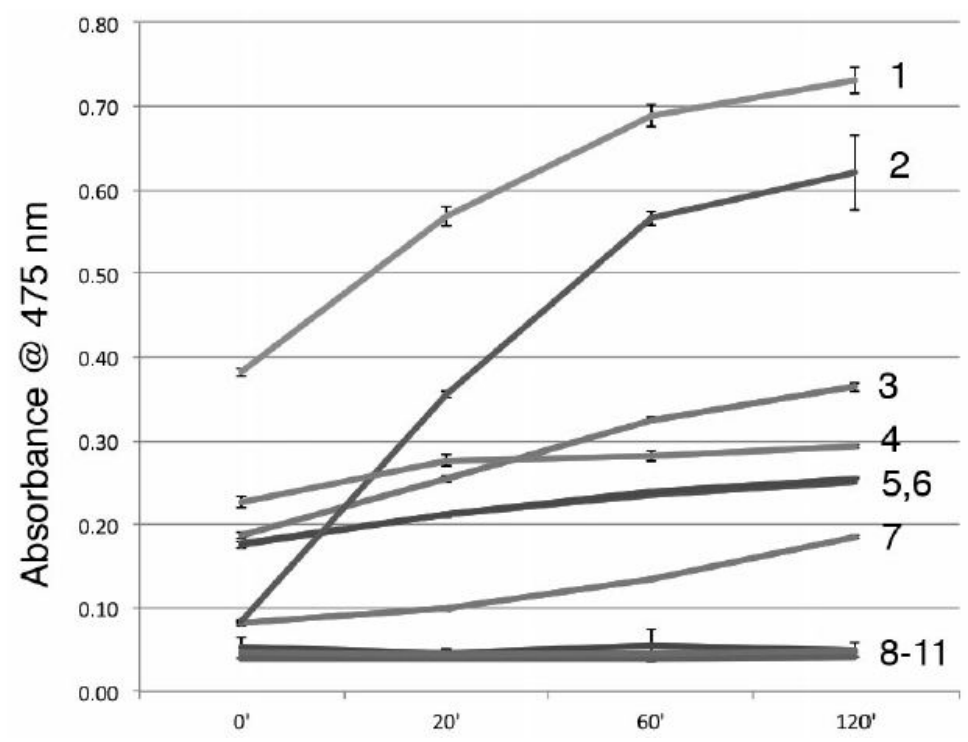

Figure 6. In vitro tyrosinase assay. Reaction containing L-tyrosine and tyrosinase with or without skin-lightening compounds were set up in triplicate in a 96-well plate. The absorbance at $475 \mathrm{~nm}$ were taken at $20^{\prime}, 60^{\prime}$ and $120^{\prime}$. Several known skin-lightening compounds (8-11) showed strong tyrosinase inhibition, as the absorbance stayed near zero, including arbutin, kojic acid (KA), PTU, and niacinamide (Nia). However, A11 (5) and MEK-I (4) did not show any inhibition of tyrosinase at all as the tyrosinase functioned like in $\mathrm{H}_{2} \mathrm{O}(6)$.

\subsection{A11 Causes Defects in Melanocyte Differentiation}

To further understand the mechanism of A11, we examined the development of melanocytes by in situ hybridization for the dct (dopachrome tautomerase) gene which is expressed in differentiated melanocytes. The result showed that the number of dct-expressing cells in A11-treated embryos is significantly lower than in the control (Figure 7A). To test whether the fewer dct-expressing cells is due to decreased proliferation of melanocyte, we performed immunostaining using the anti-phosphorylated histone $\mathrm{H} 3$ antibody. The result showed that embryos treated with A11 had lower level of proliferative melanocytes (Figure 7B). To test if A11 induced apoptosis in melanocytes, TUNEL experiments were performed and showed no significant apoptosis in A11-treated embryos (Figure 7C).
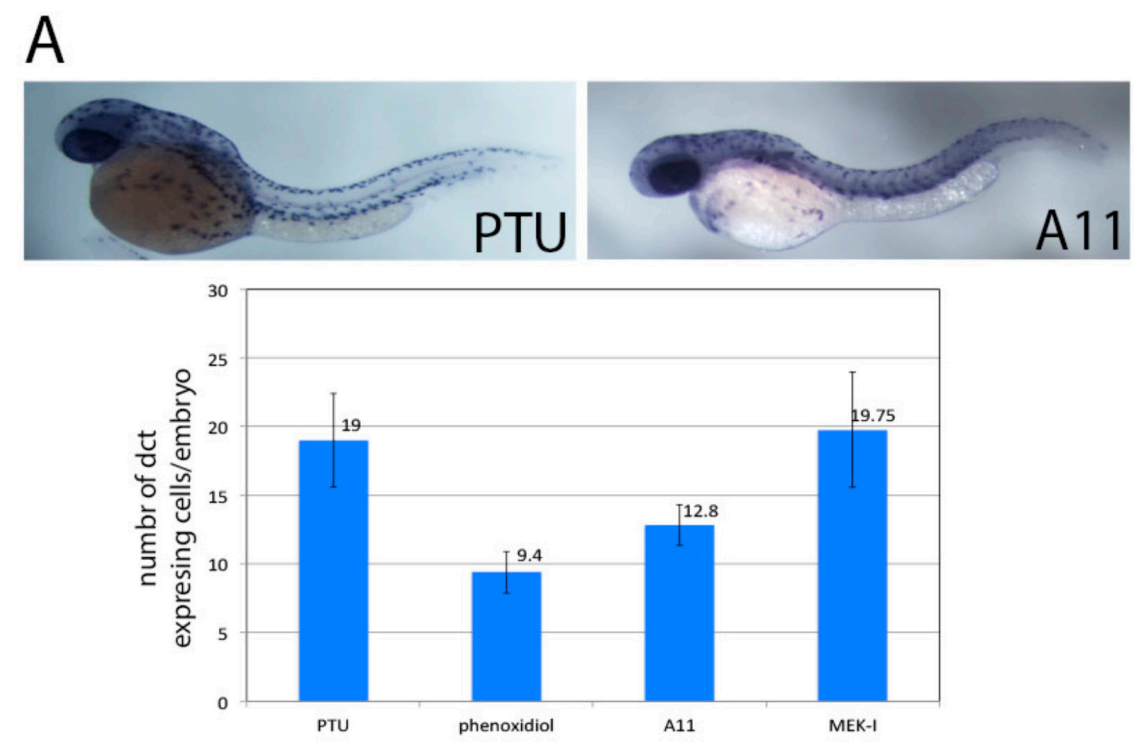

Figure 7. Cont. 

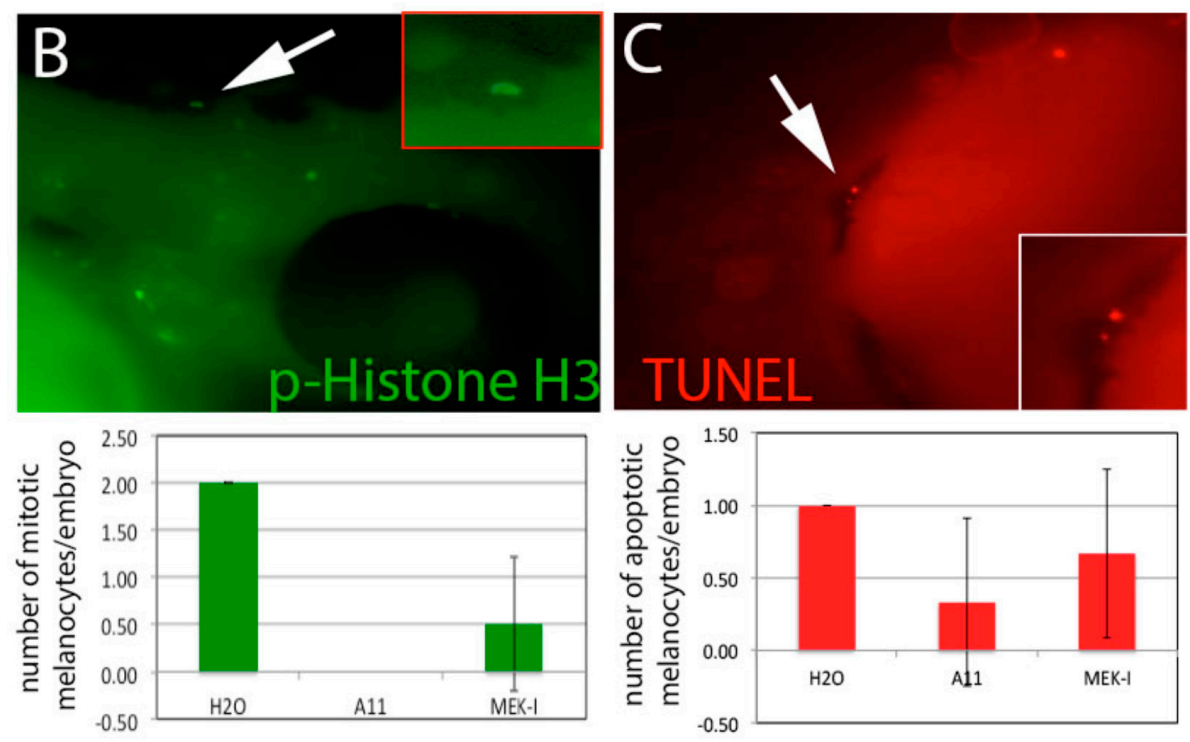

Figure 7. Inhibition of melanocyte differentiation and proliferation by A11. (A) In situ hybridization using dct gene riboprobe revealed fewer differentiated melanocytes in A11-PTU-treated embryos than those in PTU alone. The quantitative data is shown in the chart below. MEK-I has no effect on melanocyte differentiation; (B) Immunostaining using rabbit $\mathrm{mAb}$ for phosphorylated histone $\mathrm{H} 3$ (p-Histone H3) on embryos treated with A11 or MEK-I showed fewer mitotic cells. Arrow points to a melanocyte that was positive with p-Histone H3 (enlarged view in the insert). (C) TUNEL assay reveals little or no difference in the number of melanocytes between control and A11-treated embryos. Arrow points to an apoptotic melanocyte (enlarged view in the insert).

\subsection{A11 Activates MAPK and Akt Pathways in Fish Embryo}

MAPK signaling pathway has been shown to positively regulate pigment formation (Imokawa and Ishida [9]). We performed Western blotting to examine how A11 and MEK-I influence MAPK activity to regulate pigment formation. MEK-I showed expected inhibition of MAPK activity which is consistent with the current literature. To our surprise, A11 caused a distinguishably higher level of phosphorylated MAPK (activated MAPK). Another signaling pathway that is known to regulate pigment formation is Akt. Studies established that activation of Akt causes suppression of melanin synthesis in G361 melanoma cells (Oka et al. [24]). We also found that A11 slightly increases phosphorylated Akt. MEK-I, however, decreases the phosphorylated Akt (Figure 8). Note that due to the high content of lipids and proteins in the embryonic yolk, the amount of pMAPK and pAkt proteins appeared much less and the bands were fainter in the gel than we expected. However, we were able to observe a similar increase of pMAPK and pAkt by A11 using B16F10 mouse melanoma cells (Stueven et al. [17]). 


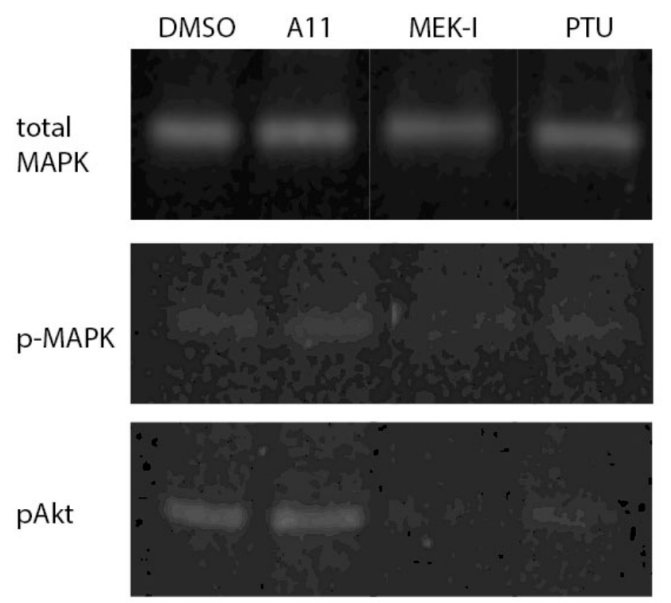

Figure 8. Activation of MAPK and Akt by A11. Western blotting using anti-total MAPK, anti-P-MPAK, or anti-Akt antibodies showed that A11 caused higher level of phosphorylated MAPK (p-MAPK) and Akt (pAkt). MEK-I inhibited MAPK activation strongly and caused inhibition of Akt. PTU showed no effect on MAPK and Akt. Total MAPK was used as an internal control which showed nearly equal amount of proteins in each lane.

\subsection{A11 Also Causes Melanin Reduction in Mammalian Cells}

Finally, we tested whether A11 has a similar drug effect on mammalian cells. We chose to use B16F10 mouse melanoma cells which are highly pigmented and metastatic cancer cells that have been widely used for skin-lightening compound test and human primary melanocytes which are derived from the epidermis of human foreskin. In both cases, we observed reduction of melanin by A11 comparable to other skin-lightening compounds (Figure 9).

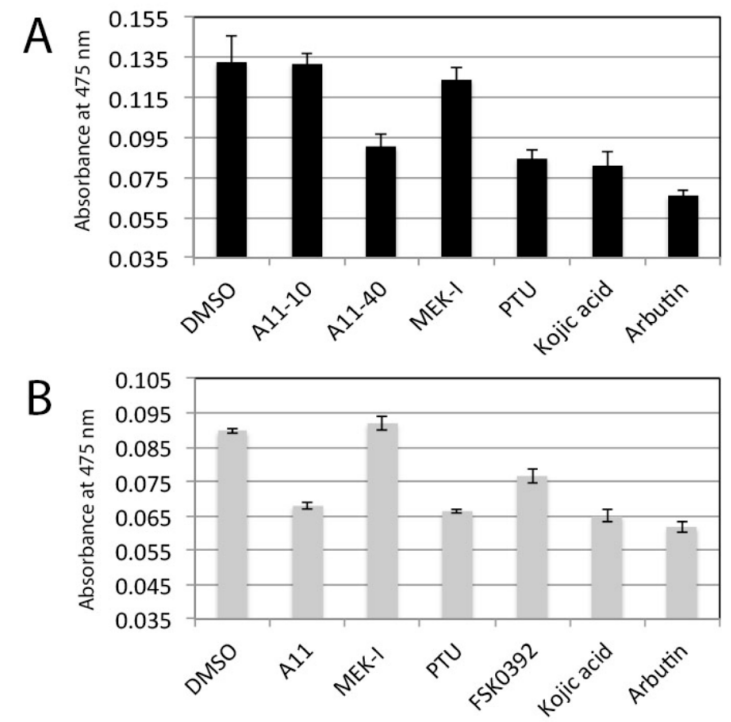

Figure 9. A11 also causes pigment reduction in mammalian melanocytes. (A) Cultured mouse melanoma cells B16F10 were treated with different skin-lightening compounds for $24 \mathrm{~h}$. The cells were lysed with $\mathrm{KOH}$ and the melanin was measured with spectrophotometer at $475 \mathrm{~nm}$. A11 showed strong reduction of melanin at $40 \mu \mathrm{M}$ (A11-40) but no reduction at $10 \mu \mathrm{M}$ (A11-10). Several skin-lightening compounds also showed strong reduction of melanin. MEK-I, however, showed no melanin reduction in B16F10 cells; (B) Normal human melanocytes were treated with skin-lightening compounds. A11, PTU, kojic acid, and arbutin showed melanin inhibition. Again, MEK-I did not show inhibition at all. FSK0392 is an A11 analog which also showed strong inhibition. 


\section{Discussion}

\subsection{A11 as a Potent and Safer Skin-Lightening Compound}

Through the comparison studies of A11 and other skin-lightening compounds, we found that A11 seems to be a potent yet safe compound for cosmetic product. A11 at $10 \mu \mathrm{M}$ inhibits almost $90 \%$ of pigment in zebrafish embryos up to 5 days post fertilization. A11 at $40 \mu \mathrm{M}$ also shows strong melanin inhibition in mammalian cells comparable to other skin-lightening compounds, such as kojic acid and arbutin. Unlike niacinamide or tretinoin, which caused strong toxicity in developing zebrafish embryos, A11 shows no detectable toxicity up to $40 \mu \mathrm{M}$. Through the late treatment experiment (Figure 4), we found that only a few skin-lightening compounds could reduce existing pigment, which included A11. In the pigment recovery experiment, A11-treated embryos recovered slower than other skin-lightening treated embryos. During our studies, we found that phenoxidiol was the other compound that could reduce existing pigment, but the phenoxidiol-treated embryos were very sick and died in a few days, with phenoxidiol toxicity being irreversible. A11 can effectively reduce melanin in mammalian cells, both in highly pigmented melanoma cells and normal human melanocytes. Together, we conclude that A11 seems superior than other skin-lightening compounds.

\subsection{Possible Cellular Mechanism of A11}

Studies show that most skin-lightening compounds reduce melanin synthesis by simply inhibiting tyrosinase enzyme activity with little toxicity on melanocytes. Those compounds, including resveratrol, could be competitive or noncompetitive inhibitor of tyrosinase. Some skin-lightening compounds, however, bind to tyrosinase which metabolizes into toxic product and kills melanocytes, such as hydroquinone, MoTP (Yang et al. [21]), and rhododendrol (Sasaki et al. [8]). A11 did not inhibit tyrosinase or cause melanocyte apoptosis, which makes A11 a very interesting skin-lightening compound. Our studies clearly show that A11 inhibits melanocyte proliferation (Figure 7). However, that cannot be the only mechanism because it does not explain how A11 can reduce existing pigment. By examining the number of differentiated melanocytes using in situ hybridization, we further discovered that A11 actually caused fewer differentiated melanocytes (Figure 7). These results together lead to an interesting model where A11 possibly causes reduction of melanocyte number by inhibiting melanocyte differentiation. If this model is correct, we anticipate a reduction of expression of melanocyte differentiation genes. Indeed, we recently published another paper using mouse melanoma cells to clearly see reduction of the Mitf and tyrosinase genes by A11 (Stueven et al. [17]).

\subsection{MAPK Signaling Pathway in Melanogenesis}

Only several skin-lightening compounds have demonstrated the ability to inhibit tyrosinase in addition to activating or inhibiting intracellular signal cascades leading to the transcriptional inhibition of melanin synthesis genes, such as haginin A (Kim et al. [10]), gallic acid (Su et al. [12]), and hydroquinone (Inoue et al. [25]). These studies focused on the MAPK and Akt signaling pathways. Unfortunately, those and our results do not quite agree with the current molecular mechanism for melanogenesis. In the review article by Imokawa and Ishida [9], UV light and $\alpha-\mathrm{MSH}$ (melanocyte stimulating hormone) induce melanin synthesis by activating MAPK and PKC pathways but haginin A and gallic acid both activate MAPK and Akt to reduce melanin (Kim et al. [10]). In our study, we show that MEK-I reduces pigment and inhibits MAPK at the same time in zebrafish embryo. A11, however, reduces pigment and activates MAPK and Akt simultaneously. One potential explanation for this is that the activation of MAPK and Akt by A11 does not directly lead to melanin synthesis, but instead there is another as-yet unidentified signaling pathway(s) involved in melanin synthesis that is regulated by A11. 
Author Contributions: Conceptualization, A.M. and C.-c.H.; Methodology, K.M., N.S., C.-c.H.; Validation, K.M, N.S., C.-c.H.; Formal Analysis, C.-c.H.; Investigation, C.-c.H.; Resources, A.M. and C.-c.H.; Data Curation, A.M. and C.-c.H.; Writing-Original Draft Preparation, C.-c.H.; Writing-Review \& Editing, C.-c.H.; Supervision, C.-c.H.; Project Administration, C.-c.H.; Funding Acquisition, C.-c.H.

Funding: This research was funded by the University of Wisconsin System grant number 106-SYS-06-8000-4 and UW-River Falls grant number [PRJ82VD].

Acknowledgments: This project was supported by a Faculty Research Grant (PRJ82VD) to C.-c.H., and Summer Research Scholarships to K.M. and N.S. from University of Wisconsin-River Falls and a Prototype Development grant (106-SYS-06-8000-4) to C.-c.H. from the University of Wisconsin System. The authors thank Michael J. Martin for comments and suggestions on the manuscript.

Conflicts of Interest: The authors declare no conflict of interest.

\section{References}

1. Battaini, G.; Monzani, E.; Casella, L.; Santagostini, L.; Pagliarin, R. Inhibition of the catecholase activity of biomimetic dinulcear copper complexes by kojic acid. J. Biol. Inorg. Chem. 2000, 5, 262-268. [CrossRef] [PubMed]

2. Jones, K.; Hughes, J.; Hong, M.; Jia, Q.; Orndorff, S. Modulation of melanogenesis by aloesin: A competitive inhibitor of tyrosinase. Pigment Cell Res. 2002, 15, 335-340. [CrossRef] [PubMed]

3. Kim, H.J.; Choi, H.-R.; Kim, D.-S.; Park, K.-C. Topical hypopigmenting agents for pigmentary disorders and their mechanisms of action. Ann. Dermatol. 2012, 24, 1-6. [CrossRef] [PubMed]

4. Brenner, M.; Hearing, V.J. Modifying skin pigmentation-approaches through intrinsic biochemistry and exogenous agents. Drug Discov. Today Dis. Mech. 2008, 5, e189-e199. [CrossRef] [PubMed]

5. Eastmond, D.A.; Rupa, D.S.; Hasegawa, L.S. Detection of hyperdiploidy and chromosome breakage in interphase human lymphocytes following exposure to the benzene metabolite hydroquinone using multicolor fluorescence in situ hybridization with DNA probes. Mutat. Res. 1994, 322, 9-20. [CrossRef]

6. Takizawa, T.; Mitsumori, K.; Tamura, T.; Nasu, M.; Ueda, M.; Imai, T.; Hirose, M. Hepatocellular tumor induction in heterozygous p53-deficient CBA mice by a 26-week dietary administration of kojic acid. Toxicol. Sci. 2003, 73, 287-293. [CrossRef] [PubMed]

7. Mezick, J.A.; Bhatia, M.C.; Capetola, R.M. Topical and systemic effects of retinoids on hor-filled utriculus size in the rhino mouse, A model to quantify "anti-keratinizing" effect of retinoids. J. Investig. Dermatol. 1984, 83, 110-113. [CrossRef] [PubMed]

8. Sasaki, M.; Kondo, M.; Sato, K.; Umeda, M.; Kawabata, K.; Takahashi, Y.; Suzuki, T.; Matsunaga, K.; Inoue, $\mathrm{S}$. Rhododendrol, a depigmentation-inducing phenolic compound, exerts melanocyte cytotoxicity via a tyrosinase-dependent mechanism. Pigment Cell Melanoma Res. 2004, 27, 754-763. [CrossRef] [PubMed]

9. Imokawa, G.; Ishida, K. Inhibitors of intracellular signaling pathways that lead to stimulated epidermal pigmentation: Perspective of anti-pigmenting agents. Int. J. Mol. Sci. 2014, 15, 8293-8315. [CrossRef] [PubMed]

10. Kim, J.H.; Baek, S.H.; Kim, D.H.; Choi, T.Y.; Yoon, T.J.; Hwang, J.S.; Kim, M.R.; Kwon, H.J.; Lee, C.H. Down regulation of melanin synthesis by haginin A and its application to in vivo lightening model. J. Investig. Dermatol. 2008, 128, 1227-1235. [CrossRef] [PubMed]

11. Lu, W.-S.; Kuan, Y.-D.; Chiu, K.-H.; Wang, W.-K.; Chang, F.-H.; Liu, C.-H.; Lee, C.-H. The extract of Rhodobacter sphaeroides inhibits melanogenesis through the MEK/ERK signaling pathway. Mar. Drugs 2013, 11, 1899-1908. [CrossRef] [PubMed]

12. Su, T.-R.; Lin, J.-J.; Tsai, C.-C.; Huang, T.-K.; Yang, Z.-Y.; Wu, M.-O.; Zheng, Y.-Q.; Su, C.-C.; Wu, Y.-J. Inhibition of melanogenesis by gallic acid: Possible involvement of the PI3K/Akt, MEK/ERK and Wnt/b-catenin signaling pathways in B16F10 cells. Int. J. Mol. Sci. 2013, 14, 20443-20458. [CrossRef] [PubMed]

13. Irion, U.; Singh, A.P.; Nusslein-Volhard, C. The developmental genetics of vertebrate color pattern formation: Lessons from zebrafish. Curr. Top. Dev. Biol. 2016, 117, 141-169. [PubMed]

14. Choi, T.-Y.; Kim, J.-H.; Ko, D.H.; Kim, C.H.; Hwang, J.-S.; Ahn, S.; Kim, S.Y.; Kim, C.-D.; Lee, J.-H.; Yoon, T.-J. Zebrafish as a new model for phenotype-based screening of melanogenic regulatory compounds. Pigment Cell Res. 2007, 20, 120-127. [CrossRef] [PubMed]

15. Garcia, G.R.; Noyes, P.D.; Tanguay, R.L. Advancements in zebrafish applications for 21st century toxicology. Pharmacol. Ther. 2016, 161, 11-21. [CrossRef] [PubMed] 
16. Huang, C.-C.; Monte, A.; Cook, J.M.; Kabir, M.S.; Peterson, K.P. Zebrafish heart failure models for the evaluation of chemical probes and drugs. Assay Drug Dev. 2013, 11, 561-572. [CrossRef] [PubMed]

17. Stueven, N.A.; Schlaeger, N.M.; Monte, A.P.; Hwang, S.-P.L.; Huang, C.-C. A novel stilbene-like compound that inhibits melanoma growth by regulating melanocyte differentiation and proliferation. Toxicol. Appl. Pharm. 2017, 337, 30-38. [CrossRef] [PubMed]

18. Westerfield, M. The Zebrafish Book; A Guide for the Laboratory Use of Zebrafish (Danio rerio); University of Oregon Press: Eugene, OR, USA, 2007.

19. Baurin, N.; Arnoult, E.; Scior, T.; Do, Q.T.; Bernard, P. Preliminary screening of some tropical plants for anti-tyrosinase activity. J. Ethnopharmacol. 2002, 82, 155-158. [CrossRef]

20. Dooley, C.M.; Schwarz, H.; Mueller, K.P.; Mongera, A.; Konantz, M.; Neuhauss, S.C.F.; Nusslein-Volhard, C.; Geisler, R. Slc45a2 and V-ATPase are regulators of melanosomal $\mathrm{pH}$ homeostasis in zebrafish, providing a mechanism for human pigment evolution and disease. Pigment Cell Melanoma Res. 2013, 26, 205-217. [CrossRef] [PubMed]

21. Yang, C.-T.; Johnson, S.L. Small molecule-induced ablation and subsequent regeneration of larval zebrafish melanocytes. Development 2006, 133, 3563-3573. [CrossRef] [PubMed]

22. Hawkins, T.A.; Cavodeassi, F.; Erdelyi, F.; Szabo, G.; Lele, A. The small molecule Mek1/2 inhibitor U0126 disrupts the chrodamesoderm to notochord transition in zebrafish. BMC Dev. Biol. 2008, 8, 42. [CrossRef] [PubMed]

23. Likhitwitayawuid, K. Stilbenes with tyrosinase inhibitory activity. Curr. Sci. 2008, 94, 44-52.

24. Oka, M.; Nagai, H.; Ando, H.; Fukunaga, M.; Matsumura, M.; Araki, K.; Ogawa, W.; Miki, T.; Sakaue, M.; Tsukamoto, K.; Konishi, H.; Kikkawa, U.; Ichihashi, M. Regulation of melanogenesis through phosphatidylinositol 3-kinase-Akt pathway in human G361 melanoma cells. J. Investig. Dermatol. 2000, 115, 699-703. [CrossRef] [PubMed]

25. Inoue, Y.; Hasegawa, S.; Yamada, T.; Date, Y.; Mizutani, H.; Nakata, S.; Matsunaga, K.; Akamatsu, H. Analysis of the effects of hydroquinone and arbutin on the differentiation of melanocytes. Biol. Pharm. Bull. 2013, 36, 1722-1730. [CrossRef] [PubMed] 